\section{OpenVAS-5: Neue Version des Werkzeugs zum Schwachstellen-Scanning und -Management}

Die ab sofort verfügbare Version OpenVAS-5, ein Werkzeug zum Schwachstellen-Scanning und -Management, umfasst eine Reihe von verbesserten und neuen Funktionen. Darunter finden sich neue Features wie Asset-Management, Delta-Reports und eingebaute SCAP-Daten. Gleichzeitig ist die Zahl der kostenfrei verfügbaren Prüfmodule auf über 25.000 gestiegen. Insgesamt sind 20 Funktionen hinzugekommen, welche vor allem die tägliche Handhabung vereinfachen sollen.

Zu den Erweiterungen zählt beispielsweise die Möglichkeit, Unterschiede zwischen zwei Scan-Ergebnissen angezeigt zu bekommen. Darüber hinaus ist die SCAP-Datenbank (Security Content-Automatisierung Protocol) mit aktuellen CPE- und CVE--Common Vulnerabilities and Exposures-Informationen direkt aus OpenVAS heraus verfügbar. Da somit nicht mehr nach Unterschieden oder Sicherheitsempfehlungen gesucht werden muss, profitieren Anwender von einer Zeitersparnis. Das neue Asset-Management ergänzt die Sicht der Scans um Prüfergebnisse zu allen IP-Geräten im Netz. OpenVAS-5 kann kostenfrei heruntergeladen werden und steht als freie Software unter der GNU GPL (General Public License). Das Bundesamt für Sicherheit in der Informationstechnik (BSI) stellte OpenVAS-5 auf dem LinuxTag im Mai 2012 in Berlin vor.

\section{Erweiterter Einsatz von OpenVAS-Technologie}

Das BSI arbeitet bei der Entwicklung mit dem Unternehmen Greenbone Networks zusammen, das OpenVAS als Basis für ihre Produktfamilie zum Schwachstellen-Scanning und -Management verwendet. Der Greenbone Security Manager (GSM) 1.7.0 wurde für Greenbone bereits als Unauthenticated Vulnerability Scanner beim amerikanischen NIST (National Institute of Standards and Technology) validiert. Weitere Informationen sind unter $h t t p: / / n v d . n i s t . g o v / v a l i-$ dation_greenbone.cfm verfügbar.

Zusammen mit den Firmen SecPod, SecuritySpace und der weiteren Community werden täglich neue Schwachstellentests entwickelt und Features verbessert. Ein OpenVAS-Bund Lizenzvertrag mit Greenbone Networks ermöglicht es Behörden, über das BSI GSM-Lizenzen, Support und weitere Leistungen günstiger oder kostenfrei zu erhalten. Das BSI und viele andere Bundesbehörden setzen OpenVAS zur Erhöhung der IT-Sicherheit in ihren Netzen ein.

\section{Cyber-Sicherheit: Mehr Schutz vor Angriffen durch Kooperation zwischen Staat und Wirtschaft}

Auf einer am 30. Mai 2012 in Bonn vom Bundesamt für Sicherheit in der Informationstechnik (BSI) ausgerichteten Fachkonferenz Cyber-Sicherheit haben sich rund 250 Teilnehmer, darunter hauptsächlich CIOs, CISOs, Geschäftsführer, IT-Leiter und IT-Sicherheitsbeauftragte aus Wirtschaft, Verwaltung und Forschung über aktuelle Trends und unterschiedliche Perspektiven der Cyber-Sicherheit informiert. In den Vorträgen, unter anderem der Beauftragten der Bundesregierung für Informationstechnik, Staatssekretärin Cornelia Rogall-Grothe, und Jörg Ziercke, Präsident des Bundeskriminalamts, stand die derzeitige Bedrohungslage ebenso im Fokus wie Kooperationsmodelle, aktuelle Lösungen und Best Practices im Bereich der Cyber-Sicherheit.
Vertreter von Staat und Wirtschaft betonten im Rahmen der Konferenz die Bedeutung einer intensivierten Zusammenarbeit, um der Bedrohung durch Cyber-Angriffe effektiv begegnen zu können. Michael Hange, Präsident des BSI, wies vor dem Hintergrund der kürzlich entdeckten Spionage-Schadsoftware „Flame“ auf die zunehmende Zahl und insbesondere die steigende Qualität der Cyber-Angriffe hin, mit denen Unternehmen, Behörden und andere Einrichtungen konfrontiert sind. „Ein Schlüssel zu einem besseren Schutz gegen Cyber-Angriffe ist die verstärkte Kooperation zwischen Staat, Wirtschaft und Wissenschaft", erklärte Hange und rief ebenso wie die Vertreter aus der Wirtschaft zu mehr gegenseitigem Vertrauen auf. „Wichtiger Ausgangspunkt aller effektiven Schutzund Abwehrmaßnahmen ist gegenseitiges Vertrauen ebenso wie ein möglichst umfassendes Lagebild. Um dies erstellen zu können, appellieren wir an betroffene Unternehmen, uns IT-Sicherheitsvorfälle zu melden."

Pilotbetrieb der Allianz für Cyber-Sicherheit gestartet Eine geeignete Plattform für die Zusammenarbeit von Staat und Wirtschaft wird das BSI im Rahmen der Allianz für Cyber-Sicherheit einrichten. Nachdem das BSI und der Branchenverband BITKOM die Allianz für Cyber-Sicherheit bereits auf der CeBIT 2012 angekündigt hatten, startet nun der Pilotbetrieb der Initiative. Als Zusammenschluss aller wichtigen Akteure im Bereich der Cyber-Sicherheit in Deutschland hat die Allianz unter anderem das Ziel, aktuelle und valide Informationen flächendeckend bereitzustellen. Die Allianz für Cyber-Sicherheit baut hierfür eine umfangreiche Wissensbasis für Teilnehmer auf und unterstützt den Informations- und Erfahrungsaustausch. Weitere Informationen sind auf der neu eingerichteten Webseite der Allianz unter https://www.allianz-fuer-cybersicherheit.de abrufbar.

\section{Call for Papers für 13. Deutschen IT-Sicherheitskongress}

Unter dem Motto „Informationssicherheit stärken - Vertrauen in die Zukunft schaffen“ findet vom 14. bis 16. Mai 2013 in Bonn der 13. Deutsche IT-Sicherheitskongress statt. Das Bundesamt für Sicherheit in der Informationstechnik (BSI) sucht ab sofort aktuelle, kreative, praxisnahe und verständliche Kongressbeiträge, die sich beispielsweise mit einem der folgenden Themenbereiche der IT-Sicherheit auseinandersetzen:

- Cyber-Sicherheit

- Information Security Management

- Sichere elektronische Identitäten

- Sicherheit in der Cloud

- Sicherheit und Mobilität

- IT-Sicherheit und Recht

- Aufklärung und Sensibilisierung

- Die Zukunft der Kryptographie: Herausforderungen in der Praxis Diese Liste ist nicht abschließend. Auch zu anderen Themen der IT-Sicherheit können interessierte Autoren aus Unternehmen, Forschungseinrichtungen, Verwaltung und anderen Institutionen bis zum 30. September 2012 per E-Mail unter papers2013@bsi.bund.de Beiträge einreichen. Die Vorschläge werden von einem hochkarätig besetzten Programmbeirat anonym begutachtet und bewertet. Die besten Einreichungen werden für den Kongress oder die im 